\title{
Abdominal wall endometriosis: an update in clinical, imagistic features, and management options
}

\author{
Mihaela Grigore ${ }^{1,2}$, Demetra Socolov', Ioana Pavaleanu', Ioana Scripcariu', \\ Ana Maria Grigore ${ }^{3}$, Romeo Micu ${ }^{4}$
}

${ }^{1}$ Department of Obstetrics and Gynecology, University of Medicine and Pharmacy "Grigore T. Popa" Iasi, ${ }^{2}$ Medis Medical Centre, Iasi, ${ }^{3}$ University of Medicine and Pharmacy "Grigore T. Popa" Iasi, ${ }^{4}$ Department of Obstetrics and Gynecology, University of Medicine and Pharmacy "Iuliu Hatieganu" Cluj-Napoca, Romania

\begin{abstract}
Abdominal wall endometriosis (AWE) is a rare condition defined by the presence of endometrial tissue in the subcutaneous fatty layer and the muscles of the abdominal wall. It is usually caused by the dissemination of endometrial tissue in the wound at the time of obstetrical and gynecological surgeries. AWE is rare and difficult to diagnose. The most frequent clinical presentation is that of a palpable subcutaneous mass near surgical scars associated with cyclic pain and swelling during menses. AWE may be an underreported pathology partly because it has scarcely received attention in the radiologic literature. Its frequency is expected to rise along with the increasing rate of cesarean deliveries; thus, it is important that physicians or sonographers are familiar with this pathology. The purpose of our review is to present the latest data regarding risk factors, clinical and imagistic findings, and management of AWE.
\end{abstract}

Keywords: abdominal wall; endometriomas; endometriosis; magnetic resonance imaging; ultrasound.

\section{Introduction}

Endometriosis is a condition characterized by the presence of uterine mucosal tissue outside the uterus. It affects $6 \%-10 \%$ of women of reproductive age being usually located in the pelvis [1]. Extra pelvic endometriosis occurs less frequently, being described almost everywhere in the body: lung, liver, gallbladder, gastrointestinal tract, perineum, central nervous system, umbilicus, inguinal hernias, or the abdominal wall. The latter may occur after surgical procedures that involve the uterine cavity, such as a cesarean delivery, allowing endometrial tissue to be transplanted. Meyer reported the first case of abdominal endometriosis in 1903 [2]. Since then, several

Received 12.09.2017 Accepted 07.10.2017

Med Ultrason

2017, Vol. 19, No 4, 430-437

Corresponding author: Mihaela Grigore, MD, PhD

University of Medicine and Pharmacy

"Grigore T.Popa" Iasi, Romania

Department of Obstetrics and Gynecology

Str. Universitatii 16, 700115, Iasi, Romania

Phone: +407 44438024

E-mail: Mihaela.grigore@edr.ro case reports or mini-series have been reported in the literature. Abdominal wall endometriosis (AWE) may be an underreported pathology, partly because it has scarcely received attention in the radiologic literature. Dissemination of information regarding AWE outside the field of obstetrics and gynecology may be useful for guiding other physicians regarding the correct therapeutic approach to adopt, thus preventing recurrence and malignant disease [3].

The purpose of our review is to present the latest data regarding risk factors, clinical findings, imagistic findings, and management of abdominal wall endometriosis.

\section{Methods}

We performed a systematic literature search of PubMed/MEDLINE, Google Scholar, and Ovid for all research articles using the terms "abdominal wall endometriosis," "abdominal wall endometriomas," "abdominal wall mass," and rectus abdominis scar" published up until May 2017. The references of those articles were then reviewed and additional publications were evaluated. 


\section{Prevalence}

It is difficult to accurately determine the prevalence of the disease in the general population. The prevalence of AWE is generally estimated to be between $0.03 \%$ and $1 \%[4,5]$. Leite et al estimate that AWE will appear in 0.03 to $3.5 \%$ of cases after obstetric procedures [3]. It is expected that its frequency will rise along with the increasing rate of cesarean deliveries being performed.

\section{Pathogenesis}

The exact etiopathogenesis of endometriosis remains controversial and several theories have been proposed including coelomic metaplasia, implantation or retrograde menstruation, vascular and lymphatic metastasis, dissemination, direct transplantation, and aerosolization [6].

Two theories have been suggested for the occurrence of AWE [7]. One theory suggests that endometrial cells may be transported to ectopic sites (particularly during surgical procedures that open the uterus). This is the most accepted theory and can explain why many patients with scar endometriosis do not have signs or a history of peritoneal endometriosis. The other theory postulates that, under the right circumstances, primitive pluripotential mesenchymal cells may undergo specialized differentiation to form endometriomas. This theory can explain the few cases of AWE reported in the literature in patients without a surgical history.

\section{Risk factors}

Almost all cases reported in the literature occur after surgery involving the uterine cavity. Very few cases of AWE reported in the literature appear without any surgery [8-11].

The majority of AWE reported has been described as being adjacent to cesarean-delivery scars [12-15]. In evaluating the obstetric history of 81 patients with endometrioma, Wicherek et al stated that cesarean delivery performed before spontaneous onset of labor may substantially increase the risk of occurrence of scar endometriomas [16]. In a recent study of a series of 34 patients with histologically proved AWE after cesarean delivery, Khan et al noted a higher BMI in women with abdominal wall endometriosis compared with controls. They hypothesized that suboptimal closure of either the uterine incision or the pelvic and abdominal layers due to the surgical difficulties encountered in obese patients may explain and support the implantation theory [17]. Sumathy et al consider that the popularisation of single-layer closure of the uterus and nonclosure of parietal and visceral peritoneum could be risk factors [18].

Although cesarean delivery and abdominal hysterectomy scars are the most common predisposing factors, AWE has also been reported in laparoscopic trocar tracts or amniocentesis needle tracts [19]. The first case of AWE after laparoscopy was reported by Healey in 1995, and since then, 15 similar cases have been reported [2022].

UAE is reported to be associated with pelvic endometriosis is reported to be between $5.3 \%$ in a study by Ding and Zhu to $14.2-26 \%$ of cases $[18,23]$.

\section{Pathology}

AWE may be confined to the superficial layers of the abdominal or pelvic wall, but it often infiltrates the deeper layers, commonly the rectus muscle. Based on the position near the abdominal wall layers, AWE can be superficial (in subcutaneous tissue only, above fascia), intermediate (infiltrating the abdominal rectus muscle fascia), and deep (in the abdominal rectus muscle, below fascia).

On gross specimen, the endometriotic tissue appears as a pinkish mass of firm consistency, and sometimes microcysts with brown material can be clearly distinguished from the surrounding yellowish subcutaneous fat [18]. When present in the muscle, AWE can also be easily distinguished due to its irregular appearance and hard consistency [18]. The literature reviewed reported that the average lesion size ranged from 2.3 to $3.2 \mathrm{~cm}$, whereas the largest lesion measured $12 \mathrm{~cm}[24,25]$.

A definitive diagnosis is made only at histopathologic analysis. Histopathologically, endometriosis can be diagnosed by the presence of endometrial glands, stroma, or hemosiderin pigment. AWE is associated with cytogenic chorion and hyperplasia of smooth muscle tissue within the soft tissue and musculature of the anterior abdominal and pelvic wall, inflammatory cells, and surrounding fibrosis [26].

\section{Diagnosis}

Typical patients with AWE present with a triad consisting of a history of cesarean delivery, cyclic pain associated with menses, and nodules near a surgical scar [27]. Physical examination is essential for an accurate diagnosis. The nodule is usually found by palpation of the subcutaneous cellular tissue, around a scar. It is located cephalad and/or lateral to the Pfannenstiel skin incision. The cephalad location occurs because the fascial incision is generally not directly below the skin incision. This 
effect is often more pronounced in patients with higher BMIs [28].

Apart from a cesarean scar, other documented locations for the tumor include 'Villar's nodule' (endometriosis in umbilical trocar scars), sterilization scars, inguinal scars, appendectomy scars, and upper abdominal scars $[7,29,30]$. Increase in the size of the lump, bleeding, and skin discoloration with cyclical changes of menstruation are not characteristically seen in all cases, but are pathognomic of scar endometriosis. Some reports consider the cyclical nature of the complaint as an important factor that predicts abdominal wall disease [31], whereas others do not consider it a universal characteristic of the disease $[32,33]$.

It is estimated that a correct diagnosis of scar endometriosis is preoperatively made in approximately $20 \%$ $50 \%$ of patients $[31,33]$. One pitfall in diagnosing AWE is that endometriosis may occur years after an operation or the nodule may not always be palpable. In some cases, the clinical aspect could be atypical, with no cyclic pain or modification of the nodule, and this might explain why its clinical recognition occurs late. In these cases, AWE can be confused with other surgical conditions. Often a general surgeon consults on AWE cases, and it is important that not only gynecologists but also general surgeons are familiar with this entity [34]

Sometimes, scar endometriosis may be incidentally discovered in women who undergo imagistic examination for other reasons.

\section{Imaging ultrasound}

Ultrasonography usually represents the first step in the evaluation of soft tissue masses. The role of ultrasound is to confirm the presence of a lesion, even if small in size, and to provide useful information regarding its size, location, margins, and structure. It is very easy with ultrasound to differentiate cystic from solid masses. However, when the mass is solid, ultrasound lacks specificity and additional diagnostic methods are necessary. When AWE is compared with other forms of endometriosis, we can say that AWE has features similar to those of deep infiltrating pelvic endometriosis [35].

Regarding the scanning technique, proper magnification is recommended of the image to reduce the field of view to $3-5 \mathrm{~cm}$ in depth [36]. It is important first to identify on a transverse or longitudinal section the normal abdominal layers of the abdominal wall far from the site where the AWE is suspected. The following layers should be identified: the hyperechoic subcutaneous tissue and the hypoechoic muscle layer covered by the thin hyperechoic abdominal fascia. The peritoneum can be identified as a thin hyperechoic line located above the intestine. The peritoneal fat located underneath the muscular layer can help in identifying the peritoneum. Then, sliding the probe while exerting slight pressure will help to locate the AWE, as a result of the pain induced [36]. Usually AWE appears on two-dimensional ultrasound as a nonhomogeneous hypoechoic mass with echogenic spots or thick echogenic strands that represent a fibrotic component in the abdominal wall (fig 1a) [24].

The margins are ill defined and there are blurred outer borders. The mass infiltrates the surrounding tissue due to an inflammatory reaction triggered by the monthly hemorrhage in this tissue [37]. A hyperechoic ring can be observed at the periphery of the nodule, and on histology, it represents edematous adipose tissue filled with cells of inflammatory origin (histiocytes and granulocytes) [36]. The echogenic patterns are dependent on the haemorrhagic and fibrous components of the lesions and on the phase of the patient's menstrual cycle [38]. Some authors consider however that the echo pattern is not always correlated with the menstrual pattern [33]. In some published cases, the ultrasound pattern is described as cystic or multicystic (fig 1b) [39]. Occasionally, a lesion appears with only punctate hypoechoic cavities in the abdominal scar [26].

The irregular shapes and borders of the endometriotic nodule appear clearer on the coronal plane of 3D ultrasound (fig 2) [40]. Three-dimensional ultrasound has proved to be very useful in recent years both in gynecology and obstetrics [41,42]. The 3D coronal plane provides a more precise analysis of the surrounding tissue and the depth of infiltration can be easily seen. 3D plays an important role in preoperative assessment by measuring the mass in all three planes and assessing whether the AWE invades the abdominal fascia. This is important in pre-

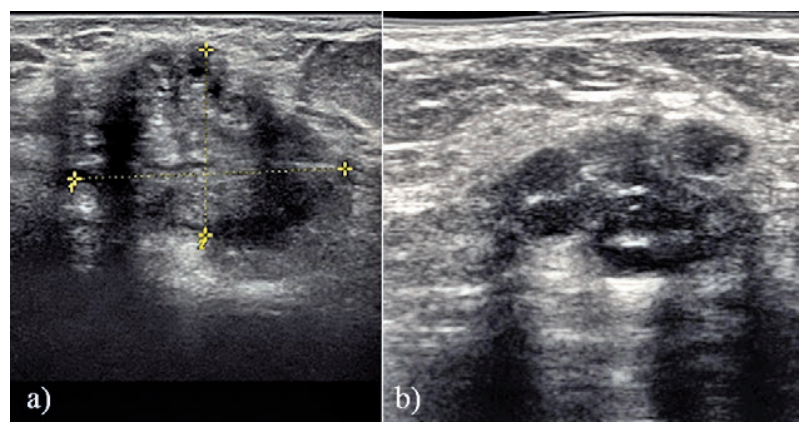

Fig 1. a) Transverse sonogram of the abdominal wall showing a 33/22-mm abdominal wall endometriosis nodule with hypoechoic content and blurred outer margins (calipers). The nodule is enclosed in the subcutaneous fatty tissue above the muscular fascia, along the scar of a previous Cesarean section; b) Transverse sonogram of the abdominal wall showing a nodule with hypoechoic content and cystic images. 
operative evaluation of the mass, especially if no other imagistic tools like CT or MRI are used. In the case of a large nodule, mesh may be needed to repair the fascia, and these procedures require adequate counselling and precise surgical planning. Therefore, exact knowledge of the AWE location is helpful in planning the surgery, choosing the reference hospital and surgical team, predicting the time of surgery, and securing additional material, such as mesh [43].

The vascular pattern can be different, from small and scanty vascularized lesions to large lesions with several vascular pedicles entering the mass from different points to abundant central vascularization (fig 3). Usually the small lesions are located in hypovascular subcutaneous fat, thus their growth is limited [44]. Doppler velocimetry can show intralesional vascularization if the tumor size is greater than $15 \mathrm{~mm}$ [44]. The presence of central vascularity is a highly determinative finding of malignancy for soft tissue lesions, according to Fleischer et al [45]. Both clinicians and radiologists should consider, in the presence of a rapidly growing, painless mass and the absence of previous surgery, malignant soft tissue masses in the abdominal wall as a differential diagnosis.

Elastography is a novel ultrasound-based imaging modality that assesses the elasticity of visualized tissues. One of the many elastography methods is strain elastography, based on the phenomenon that after applying pressure with the probe the strain of hard tissues is lower than that of soft tissues. Gradient values of strain are visualized on a color map, representing the stiffness of the examined area [43]. Wozniak et al showed that elastography improves the performance of B mode ultrasound in the preoperative assessment of AWE location. Moreover, the accuracy of alpha-blend elastography in the preoperative assessment of AWE location is not decreased in overweight and obese patients [43]

All the described ultrasound findings are nonspecific, and a wide spectrum of disorders that may result in a mass in the abdominal wall should be considered in the imaging differential diagnosis. This includes primary or metastatic tumor, desmoid tumor, lymphoma, and non-neoplastic causes, such as suture granuloma, ventral hernia, hematoma, abscess, or retained surgical material $[8,46]$. Sonography may exclude granuloma, hernia, hematoma, abscess, or seroma in view of the solid appearance and vascular nature of AWE [24].

Acute hematomas are typically highly echogenic whereas seromas and resolving hematomas are hypoechoic or anechoic. Abscess demonstrates an air-fluid level. Correlation with clinical history is important, because both hematomas and abscesses manifest in the

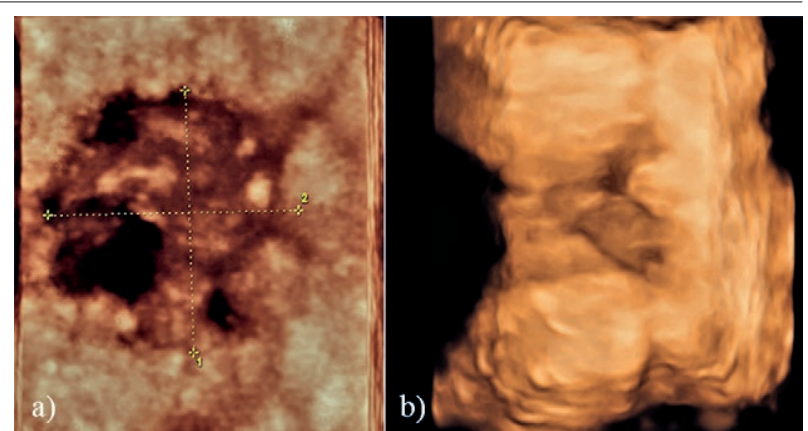

Fig 2. a) The reconstructed coronal plane with $3 \mathrm{D}$ ultrasound shows the heterogeneous mass with irregular and speculated margins; b) The reconstructed coronal plane with 3D ultrasound shows the heterogeneous mass with irregular and speculated margins and cystic images inside of the tumor.

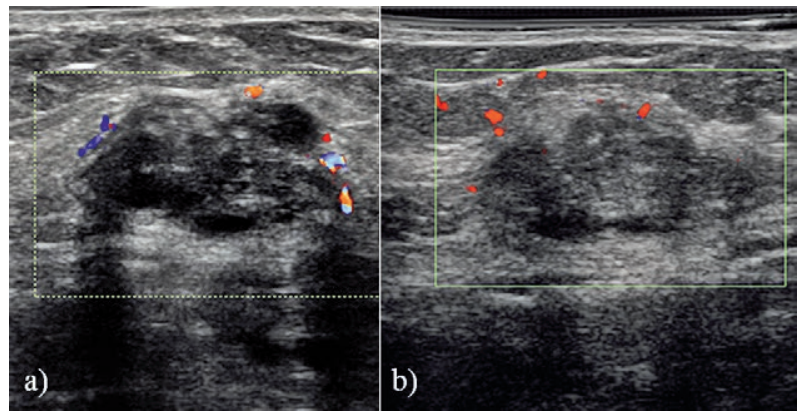

Fig 3. Color Doppler US images showing peripheral flow within the mass ( $a$ and $b$ ).

setting of a recent surgery or procedure [47]. Lymphoceles are usually anechoic and have septa. A granuloma on ultrasound may appear as irregular and inhomogeneous, partly hyper- and partly hypoechoic and is difficult to characterize at imaging alone and may require biopsy [47].

Difficulties can appear in the differential diagnosis with desmoid tumours. Abdominal wall desmoid tumours are rare, slow-growing, benign, muscular-aponeurotic fibrous tumours with the tendency to locally invade and recur. Desmoid tumours lack a capsule and are infiltrative. Therefore, although histologically benign, they are locally aggressive. Typically, desmoid tumours do not manifest with cyclical pain imaging, and clinical findings must be closely correlated because the ultrasound appearance of a desmoid tumour may closely resemble that of scar endometriosis. On ultrasound, desmoid tumours are usually small and hypoechoic with well-defined margins (fig 4) [48].

Metastases usually present on ultrasound as poorly defined hypoechoic masses with increased vascularity. In lymphoma, ultrasound findings are often nonspecific, and the appearances are variable, including a large mass, 


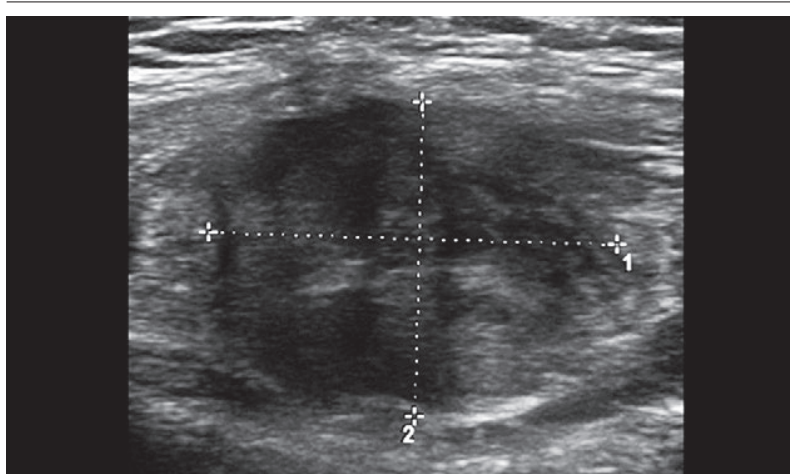

Fig 4. Abdominal desmoid tumor - hypoecoic nodule with regular contour.

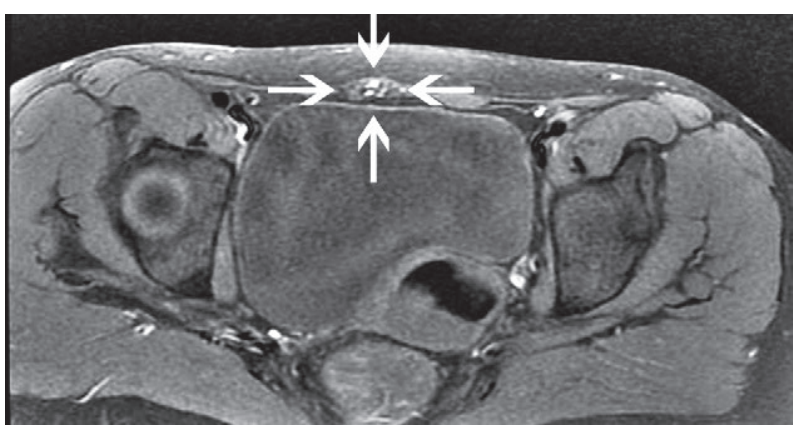

Fig 5. MRI- Axial T1- enhanced T1-weighted fat-sup $\neg$ pressed showing a mass (white arrows) within the anterior abdominal wall abutting the right rectus abdominis muscle with foci of hyperintensity suggesting haemorrhage.

nodal or confluent nodal structures, small nodules $(<1$ $\mathrm{cm})$, and disseminated myositis and panniculitis [49].

\section{Magnetic Resonance Imaging}

MRI is typically considered the best method for describing the anatomy of a soft tissue mass and its surrounding structures. MRI imaging is preferred in younger patients, because of its improved tissue characterization and lack of ionizing radiation. It can detect even very small lesions and has the advantage of a clearer depiction of the delineation between muscles and abdominal subcutaneous tissues and infiltration of abdominal and pelvic wall structures [26]. MRI can determine the location and depth of infiltration in the surrounding tissues, information useful in choosing the best method for closing the fascia defect during surgery.

AWE appears as a hyperintense heterogeneous nodule associated with a surgical scar on both T1- (with and without fat suppression) and T2-weighted images, a result of subacute hemorrhage within the endometriotic crypts although this finding may vary $[26,38,50]$ ( fig 5). MRI imaging is capable of showing the presence of hemorrhage in the abdominal wall mass and can provide information on the chronicity of a hematoma associated with scar endometriosis or other processes [47]. A feeding vessel also occasionally can be seen. Alternatively, scar endometriosis may be isointense relative to muscle on T1-weighted images and may be difficult to identify [33]. In cases with chronic scar endometriosis, lesions may have spiculated margins and low signal intensity on T2-weighted images, because of both dominant fibrotic and hemosiderotic components [26,47].

However in AWE, the signal characteristics and contrast enhancement pattern of AWE are not specific, and the true histological nature of soft-tissue masses often cannot be made, with a few exceptions (eg, lipoma or haemorrhage) [46,51].

\section{Computerized tomography}

Computerized tomography (CT) involves irradiation and requires an intravenous contrast agent. On CT AWE typically appears as a solid soft-tissue mass directly associated with an area of surgical scarring [47]. The findings depend on the phase of the menstrual cycle, the proportion of stromal and glandular elements, the amount of bleeding, and the degree of inflammatory and fibrotic response. It may be hyper attenuating compared with muscle, although its attenuation may vary $[37,52$.]. Usually, mild to moderate enhancement is seen after administration of intravenous contrast material $[24,47]$. Feeding vessels may be seen within or adjacent to the mass. However, it may be difficult to distinguish scar endometriosis from bland scarring and other processes at unenhanced and contrast-enhanced CT; thus, correlation of clinical and imaging findings is important [26].

The different imaging modalities (ultrasound, MRI, CT) are nonspecific but useful in determining the extent of disease and assist in the planning of operative resection, especially in recurrent and large lesions [53].

\section{Fine needle aspiration}

Ultrasound-guided fine needle aspiration (FNA) is an accurate, inexpensive diagnostic procedure in women with abdominal wall masses, showing the difference between benign and malignant conditions. It has been reported to diagnose endometrioma in isolated cases [54-58]. FNA is easy to perform because the nodule is firm [59]. With FNA, clusters can be identified of epithelial endometrial-like cells, endometrial-like stromal cells, and haemosiderin-laden macrophages in the lesion. Sometimes because of the limited amount of sample material as well as the presence of fibrotic tissue in the old 
lesions of endometriosis, the diagnosis on FNA may be inconclusive and an additional histologic biopsy may be considered [11].

However, FNA use is controversial because of the risk of causing new implants at the puncture sites [27]. To avoid the spread of endometriosis after these procedures, it is advisable to include the site of aspiration in the field of operative resection [53].

\section{Risk of malignization}

Malignant change in AWE is rare and is reported in only about $1 \%$ of cases [24]. The principal risk factors of malignant transformation of endometriosis include advanced age of the patient, if they are postmenopausal, and if the tumor diameter of an endometriotic lesion is $9 \mathrm{~cm}$ [60]. Malignancy of AWE may occur from just a few months until up to 18 years after surgery [24]. Clear cell carcinoma is the most common histological subtype, and the 20 -month survival rate is only $57 \%$ [61]. Several other types have been sporadically reported in the literature, including carcinosarcoma, cystadenocarcinoma, and serous papillary carcinoma [62-64]. The main prevention method is represented by wide excision of AWE with clear margins.

\section{Management}

Some studies have reported the use of medical treatment (oral contraceptives, gonadotropin-releasing hormones, danazol, and progesterone) though with a low rate of success and often followed by recurrence after cessation of the drug [31].

Surgery represents the treatment of choice for AWE because it offers the best chance for both definitive diagnosis and treatment. It is important to remove the nodule and the adjacent fascia completely; otherwise, AWE can reoccur or new lesions can form if the neighbouring parenchyma is inoculated during the operation [31]. In cases of small nodules, which are present in most cases, superficially located surgery can be relatively easy. In several cases, the excision may be technically difficult and the surgical mass excised may need to be enlarged if the lesion extends to deeper tissues, such as aponeurosis, muscle, or more rarely, peritoneum. In these cases, sometimes a mesh or aponeurotic muscle flap for covering the defect is needed [65]. Surgical resection leads to healing in $95 \%$ of cases, and relapses occur in the rest of the cases [8]. Usually a high rate of recurrence $(9.1 \%)$ is related to a previous inadequate resection of the endometriotic lesion [31]. The margins should be at least $1 \mathrm{~cm} \mathrm{[18].}$
Although all authors recommend adequate surgical excision for favourable outcomes, no studies have addressed whether the size of the surgical margin affects the recurrence rate.

\section{Prevention}

Because scar endometrioma mainly originates during cesarean delivery, it is important to reevaluate the best practices and care related to this procedure. Several methods are recommended to avoid the incidence of AWE, including handling uterine tissue very gently, ensuring meticulous control of bleeding, washing of the intraabdominal cavities before closure of the abdomen, and avoiding subcutaneous dead spaces [66]. Sumathy et al recommend exteriorisation of the uterus for suturing, leaving alone the endometrium while suturing, strict irrigation of the abdominopelvic cavity, approximating the visceral and parietal peritoneum, and using separate needles for uterine and abdominal wall suturing [18]. Up to now, no data suggest that these strategies can prevent the occurrence of scar endometriosis. New studies are required to determine which prophylactic measures are most efficient.

\section{Conclusion}

AWE is a condition that generally, but not exclusively, occurs in patients who have a palpable mass associated with pain and a previous pelvic surgery. Combination of 2D, 3D, and Doppler sonography in correlation with clinical data and patient history (particularly the occurrence of previous surgery adjacent to the lesion) may aid considerably in making the correct diagnosis of AWE. The different imaging modalities (ultrasound, MRI, CT) are nonspecific but useful in determining the extent of disease and assist in the planning of operative resection, especially in recurrent and large lesions.

Although rare, it is important that physicians or sonographers are familiar with this pathology. Considering the increasing rate of caesarean deliveries, it is expected that this pathology will be encountered more frequently in daily practice.

\section{Conflict of interest: none}

\section{References}

1. Burney RO, Giudice LC. Pathogenesis and pathophysiology of endometriosis. Fertil Steril 2012;98:511-519.

2. Meyer R. Adenomatous proliferation of the serosa in an abdominal scar. Z Geburtsh Gynak 1903;49:32-41. 
3. Leite GK, De Carvalho LF, Korkes H, Guazzelli TF, Kenj G, Viana Ade T. Scar endometrioma following obstetric surgical incisions: retrospective study on 33 cases and review of the literature. Sao Paulo Med J 2009;127:270-277.

4. Firilas A, Soi A, Max M. Abdominal incision endometriomas. Am Surg 1994;60:259-261.

5. Bergqvist A. Different types of extragenital endometriosis: a review. Gynecol Endocrinol 1993;7:207-221.

6. Chang Y, Tsai EM, Long CY, Chen YH, Kay N. Abdominal wall endometriomas. J Reprod Med 2009;54:155-159.

7. Steck WD, Helwig EB. Cutaneous endometriosis. JAMA 1965;191:167-170.

8. Horton JD, Dezee KJ, Ahnfeldt EP, Wagner M. Abdominal wall endometriosis: a surgeon's perspective and review of 445 cases. Am J Surg 2008;196:207-212.

9. Al Shakarchi J, Bohra A. Endometrioma in a virgin abdomen masquerading as an intramuscular lipoma. J Surg Case Rep 2015;2015:rjv023.

10. Ideyi SC, Schein M, Niazi M, Gerst PH. Spontaneous endometriosis of the abdominal wall. Dig Surg 2003;20:246248.

11. Dwivedi AJ, Agrawal SN, Silva YJ. Abdominal wall endometriomas. Dig Dis Sci 2002;47:456-461.

12. Khamechian T, Alizargar J, Mazoochi T. 5-Year data analysis of patients following abdominal wall endometrioma surgery. BMC Womens Health 2014;14:151.

13. Vellido-Cotelo R, Muñoz-González JL, Oliver-Pérez $\mathrm{MR}$, et al. Endometriosis node in gynaecologic scars: a study of 17 patients andthe diagnostic considerations in clinical experience in tertiary care center. BMC Womens Health 2015;15:13.

14. Ecker AM, Donnellan NM, Shepherd JP, Lee TT. Abdominal wall endometriosis: 12 years of experience at a large academic institution. Am J Obstet Gynecol 2014;211:363. e1-5.

15. Oh EM, Lee WS, Kang JM, Choi ST, Kim KK, Lee WK. A Surgeon's perspective of abdominal wall endometriosis at a caesarean section incision: Nine cases in a single institution. Surg Res Pract 2014;2014:765372.

16. Wicherek L, Klimek M, Skret-Magierlo J, et al. The obstetrical history in patients with Pfannenstiel scar endometriomas- an analysis of 81 patients. Gynecol Obstet Invest 2007;63:107-113.

17. Khan Z, Zanfagnin V, El-Nashar SA, Famuyide AO, Daftary GS, Hopkins MR. Risk factors, clinical presentation, and outcomes for abdominal wall endometriosis. J Minim Invasive Gynecol 2017;24:478-484.

18. Sumathy S, Mangalakanthi J, Purushothaman K, Sharma D, Remadevi C, Sreedhar S. Symptomatology and Surgical Perspective of Scar Endometriosis:A Case Series of 16 Women. J Obstet Gynaecol India 2017;67:218-223.

19. Kaunitz A, Di Sant'Agnese PA. Needle tract endometriosis: an unusual complication of amniocentesis. Obstet Gynecol 1979;54:753-755.

20. Vuksic T, Rastovic P, Dragisic V. Abdominal Wall Endometrioma after Laparoscopic Operation of Uterine Endometriosis. Case Rep Surg 2016;2016:5843179.
21. Emre A, Akbulut S, Yilmaz M, Bozdag Z. Laparoscopic trocar port site endometriosis: a case report and brief literature review. Int Surg 2012;97:135-139.

22. Chmaj-Wierzchowska K, Pieta B, Czerniak T, Opala T. Endometriosis in a post-laparoscopic scar-case report and literature review. Ginekol Pol 2014;85:386-389.

23. Ding Y, Zhu J. A retrospective review of abdominal wall endometriosis in Shanghai, China. Int J Gynaecol Obstet 2013;121:41-44.

24. Hensen JH, Van Breda Vriesman AC, Puylaert JB. Abdominal wall endometriosis: clinical presentation and imaging features with emphasis on sonography. AJR Am J Roentgenol 2006;186:616-620.

25. Minaglia S, Mishell DR Jr, Ballard CA. Incisional endometriomas after Cesarean section: a case series. J Reprod Med 2007;52:630-634.

26. Balleyguier C, Chapron C, Chopin N, Hélénon O, Menu Y. Abdominal wall and surgical scar endometriosis: results of magnetic resonance imaging. Gynecol Obstet Invest 2003;55:220-224.

27. Esquivel-Estrada V, Briones-Garduño JC, MondragónBallesteros R. Endometriosis implant in cesarean section surgical scar. Cir Cir 2004;72:113-115.

28. Rindos NB, Mansuria S. Diagnosis and management of abdominal wall endometriosis: a systematic review and clinical recommendations. Obstet Gynecol Surv 2017;72:116122.

29. Victory R, Diamond MP, Johns DA. Villar's nodule: a case report and systematic literature review of endometriosis externa of the umbilicus. J Minim Invasive Gynecol 2007; 14:23-32.

30. Harry VN, Shanbhag S, Lyall M, Narayansingh GV, Parkin DE. Isolated clear cell adenocarcinoma in scar endometriosis mimicking an incisional hernia. Obstet Gynecol 2007;110:469-471.

31. Bektaş H, Bilsel Y, Sari YS, et al. Abdominal wall endometrioma; a 10-year experience and brief review of the literature. J Surg Res 2010;164:e77-e81.

32. Chatterjee SK. Scar endometriosis: a clinicopathologic study of 17 cases. Obstet Gynecol 1980;283:81-84.

33. Blanco RG, Parithivel VS, Shah AK, Gumbs MA, Schein M, Gerst PH. Abdominal wall endometriomas. Am J Surg 2003;185:596-598.

34. Akbulut S, Sevinc MM, Bakir S, Cakabay B, Sezgin A. Scar endometriosis in the abdominal wall: a predictable condition for experienced surgeons. Acta Chir Belg 2010;110:303-307.

35. Hudelist G, Ballard K, English J, et al. Transvaginal sonography vs. clinical examination in the preoperative diagnosis of deep infiltrating endometriosis. Ultrasound Obstet Gynecol 2011;37:480-487.

36. Savelli L, Manuzzi L, Di Donato N, et al. Endometriosis of the abdominal wall: ultrasonographic and Doppler characteristics. Ultrasound Obstet Gynecol 2012;39:336-340.

37. Francica G, Giardiello C, Angelone G, Cristiano S, Finelli R, Tramontano G. Abdominal wall endometriomas near cesarean delivery scars: sonographic and color dop- 
pler findings in a series of 12 patients. J Ultrasound Med 2003;22:1041-1047.

38. Wolf Y, Haddad R, Werbin N, Skornick Y, Kaplan O. Endometriosis in abdominal scars: a diagnostic pitfall. Am Surg 1996;62:1042-1044.

39. Morales Martinez C, Tejuca Somoano S. Abdominal wall endometriosis. Am JL Obstet Gynecol 2017. doi:10.1016/j. ajog.2017.07.033.

40. Picard A, Varlet MN, Guillibert F, et al. Three-dimensional sonographic diagnosis of abdominal wall endometriosis: a useful tool? Fertil Steril 2011;95:289.e1-4.

41. Grigore M, Iliev G, Gafitanu, Cojocaru C. The fetal abdominal wall defects using 2D and 3D ultrasound. Pictorial essay. Med Ultrason 2012;14:341-347.

42. Grigore M, Iliev G. Diagnosis of sacrococcygeal teratoma using two and three-dimensional ultrasonography: two cases reported and a literature review. Med Ultrason 2014;16:274-277.

43. Wozniak S, Czuczwar P, Szkodziak P, et al. Elastography improves the accuracy of ultrasound in the preoperative assessment of abdominal wall endometriosis. Ultraschall Med 2015;36:623-629.

44. Francica G, Scarano F, Scotti L, Angelone G, Giardiello C. Endometriomas in the region of a scar from Cesarean section: sonographic appearance and clinical presentation vary with the size of the lesion. J Clin Ultrasound 2009;37:215220.

45. Fleischer AC, Milam MR, Crispens MA, Shappell HW. Sonographic depiction of intratumoral vascularity with 2- and 3-dimensional color Doppler techniques. J Ultrasound Med 2005;24:533-537.

46. Francica G. Reliable clinical and sonographic findings in the diagnosis of abdominal wall endometriosis near cesarean section scar. World J Radiol 2012;28:135-140.

47. Gidwaney R, Badler RL, Yam BL, et al. Endometriosis of abdominal and pelvic wall scars: multimodality imaging findings, pathologic correlation, and radiologic mimics. Radiographics 2012;32:2031-2043.

48. Koshariya M, Shukla S, Khan Z, et al. Giant desmoid tumor of the anterior abdominal wall in a young female: a case report. Case Rep Surg 2013;2013:780862.

49. Ahn SE, Park SJ, Moon SK, Lee DH, Lim JW. Sonography of Abdominal Wall Masses and Masslike Lesions: Correlation With Computed Tomography and Magnetic Resonance Imaging. J Ultrasound Med 2016;35:189-208.

50. Yu CY, Perez-Reyes M, Brown JJ, Borrello JA. MR appearance of umbilical endometriosis. J Comput Assist Tomogr 1994;18:269-271.

51. Onbas O, Kantarci M, Alper F, et al. Nodular endometriosis: dynamic MR imaging. Abdom Imaging 2007;32:451-456.
52. Haim N, Shapiro-Feinberg M, Zissin R. Incisional endometriomas: CT findings. Emerg Radiol 2005;11:162-163.

53. Solak A, Genç B, Yalaz S, Sahin N, Sezer TÖ, Solak I. Abdominal wall endometrioma: ultrasonographic features and correlation with clinical findings. Balkan Med J 2013;155-160.

54. Griffin JB, Betsill WL Jr. Subcutaneous endometriosis diagnosed by fine needle aspiration cytology. Acta Cytol 1985;29:584-588.

55. Pikoulis E, Karavokiros J, Veltsista K, et al. Abdominal scar endometriosis after caesarean section: report of five cases. West Indian Med J 2011;60:351-353.

56. Catalina-Fernández I, López-Presa D, Sáenz-Santamaria J. Fine needle aspiration cytology in cutaneous and subcutaneous endometriosis. Acta Cytol 2007;51:380-384.

57. Fulciniti F, Caleo A, Lepore M, Fortunato A, Vetrani A, Palombini L. Fine needle cytology of endometriosis: experience with 10 cases. Acta Cytol 2005;49:495-499.

58. Kim JY, Kwon JE, Kim HJ, Park K. Fine-needle aspiration cytology of abdominal wall endometriosis: a study of 10 cases. Diagn Cytopathol 2013;41:115-119.

59. Pachori G, Sharma R, Sunaria RK, Bayla T. Scar endometriosis: Diagnosis by fine needle aspiration. J Cytol 2015;32:65-67.

60. Kobayashi H, Sumimoto K, Moniwa N, et al. Risk of developing ovarian cancer among women with ovarian endometrioma: 391 a cohort study in Shizuoka, Japan. Int J Gynecol Cancer 2007; 17:37-43.

61. Sergent F, Baron M, Le Cornec JB, Scotté M, Mace P, Marpeau L. Malignant transformation of abdominal wall endometriosis: a new case report. J Gynecol Obstet Biol Reprod (Paris) 2006;35:186-190.

62. Leng J, Lang J, Guo L, Li H, Liu Z. Carcinosarcoma arising from atypical endometriosis in a cesarean section scar. Int $\mathrm{J}$ Gynecol Cancer 2006;16:432-435.

63. Matter M, Schneider N, McKee T. Cystadenocarcinoma of the abdominal wall following caesarean section: case report and review of the literature. Gynecol Oncol 2003;91:438443.

64. Fargas Fàbregas F, Cusidó Guimferrer M, Tresserra Casas F, Baulies Caballero S, Fábregas Xauradó R. Malignant transformation of abdominal wall endometriosis with lymph node metastasis: Case report and review of literature. Gynecol Oncol Case Rep 2014;8:10-13.

65. Vaz-de-Macedo C, Gomes-da-Costa A, Mendes S, et al. Abdominal wall endometriosis excision with mesh closure - report of two cases. Surg Technol Int 2016;28:196-201.

66. Ozel L, Sagiroglu J, Unal A, et al. Abdominal wall endometriosis in the cesarean section surgical scar: a potential diagnostic pitfall. J Obstet Gynecol Res 2012;38:526-530. 